\title{
Fabrication of High Array Zinc-Indium Oxide Nanowires and a Nanowire Gas Sensor
}

\author{
Chin-Guo Kuo, Jeng-Ji Huang, ${ }^{1}$ Jung-Hsuan Chen, ${ }^{*}$ and Ren-Jun Zeng \\ Department of Industrial Education, National Taiwan Normal University, Taipei 10610, Taiwan \\ 162, Sec. 1, Heping E. Rd., Taipei 10610, Taiwan \\ ${ }^{1}$ Department of Electrical Engineering, National Taiwan Normal University, \\ 162, Sec. 1, Heping E. Rd., Taipei 10610, Taiwan
}

(Received September 30, 2016; accepted January 6, 2017)

Keywords: zinc-indium oxide, nanowires, anodic aluminum oxide, die casting, gas sensing

In this research, we used anodic aluminum oxide (AAO) nanoporous membranes to produce high-density and high-regularity zinc-indium oxide nanowires, and in the process, the production control parameters could be manipulated to adjust the diameter and distribution of the nanopores on the AAO membranes to produce one-dimensional zinc-indium oxide nanowires with high aspect ratios. A vacuum die casting method was used to fill the alloy into the high aspect ratio holes to form nanowires. The prepared nanowires were soaked in sodium hydroxide solution for wet etching to remove AAO membrane. Then, the nanowires were subjected to oxidation treatment to become zinc-indium oxide nanowires, so as to increase the detectable area of the metal oxide semiconductor sensor for carbon monoxide gas as well as to plate the electrode finishing components. In the experiment, the zinc-indium bulk was subjected to differential scanning calorimetry (DSC) to determine its melting point. Scanning electron microscopy (SEM) was used for the microstructural analysis of the zinc-indium nanowires. Finally, the gas sensing properties of the zinc-indium oxide nanowires were tested by exposing the device to carbon monoxide gas and determining its sensitivity and response time.

\section{Introduction}

Lately there has been no significant decrease in death toll due to carbon monoxide poisoning. Relevant safety regulations have become more stringent, and high-performance carbon monoxide detectors are receiving increasing attention. Gas sensors made of metal oxide semiconductors (MOSs) are heat- and corrosion-resistant and are characterized by a fast response rate and easily made components. Therefore, they are widely used in household and factory environments to detect poisonous and flammable gases. ${ }^{(1-3)}$ The principle of metal oxide semiconductor gas sensors is to use a heater to maintain a constant temperature, and then, when the metal oxide comes into contact with the detected gas, the change in resistance is used to determine the gas concentration. Traditional semiconductor gas sensors are mostly thin films or the diode type, whose gas absorption area is very limited. Product miniaturization further reduces the contact surface area, leading to decreased sensitivity in gas detection. Compared with the traditional semiconductor sensors, gas sensors composed of nanomaterials have a larger depth-to-width ratio and a higher surface area *Corresponding author: e-mail: jhchen@ntnu.edu.tw http://dx.doi.org/10.18494/SAM.2017.1535 
ratio and are capable of increasing the contact area with the gas to be detected to achieve better sensitivity and response speed plus better energy efficiency. Therefore, nanomaterials have been a frequent topic in research studies and discussions of gas sensors. ${ }^{(4-10)}$

In nanomaterials, the one-dimensional structure is the most common structure, including products such as nanotubes, nanowires, and nanocolumns. In this research, we used an electrochemical method to produce AAO membranes and a vacuum die-casting method to fabricate one-dimensional zinc-indium nanowires. Taking advantage of nanowires, a high surface area ratio for gas sensing was the focus of this research. Moreover, in this work, we proposed an alternative method of producing zinc-indium oxide nanowires that would be cheaper than other vacuum processes. The nanowire arrays produced can be of equal length, stable, and regularly aligned. In the future, with micro-electromechanical technology, nanowire sensors can be mass produced and applied to medicine, biotechnology, automobiles, and environmental detection.

\section{Materials and Methods}

In this research we used a porous alumina membrane as the substrate, which was covered with a layer of molten zinc-indium metal bulk; a forward casting force was applied to inject the molten metal into the nanopores of the template. Then, the aluminum substrate and alumina template were removed and the nanowires were put in an atmosphere furnace for heat treatment and oxidation into zinc-indium oxide nanowires. Finally, the nanowires were plated with gold films as electrodes. The production flow is illustrated in Fig. 1.

\subsection{Preparing an array-type porous alumina membrane}

In this research, we used a $99.7 \%$ pure aluminum plate as the substrate, a well-mixed perchloric acid, ethanol, and monobutyl ether glycol solution in a 3:14:3 ratio as the electrolytic polishing solution, a pure aluminum plate as the anode, and a graphite plate as the cathode for electrolytic

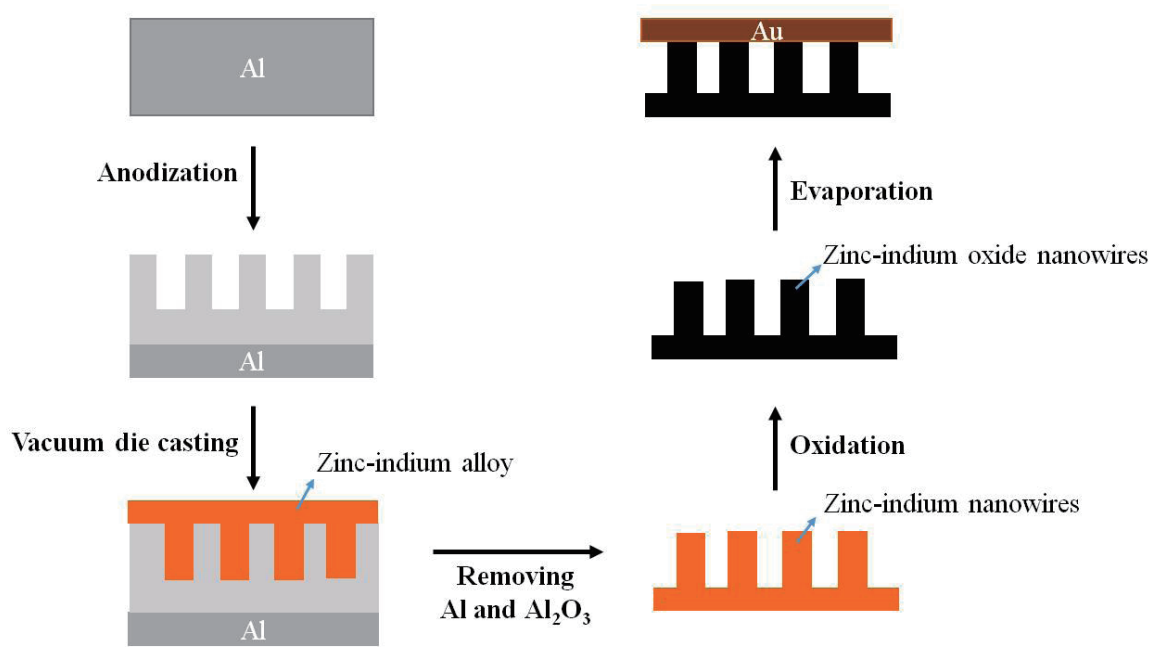

Fig. 1. (Color online) Process flow for sensor components. 
polishing to produce an aluminum plate with a smooth surface. Then, a $0.3 \mathrm{M}$ oxalic acid solution was used to conduct a two step anodic treatment with the aluminum plate as the anode and the graphite plate as the cathode. The first step was a 1-h anodic treatment at $40 \mathrm{~V}$, followed by removing the alumina membrane from the aluminum plate with $6 \%$ phosphoric acid solution and $2 \%$ chromic acid solution below $80{ }^{\circ} \mathrm{C}$. The second step was the same as the anodic treatment but lasted $6 \mathrm{~h}$. If the control factors, such as applied voltage, anode processing time, surface roughness of aluminum pieces, and electrolyte temperature and concentration, are appropriately adjusted, the pores can be regularly aligned with consistency. The prepared template in this work was an alumina membrane with $80 \mathrm{~nm}$-diameter pores.

\subsection{Producing zinc-indium oxide nanowires}

A vacuum die casting method is used to fabricate metallic nanowires. In the casting process, the molten metal is injected into the anodic alumina membrane by a hydraulic force forming metallic nanowires. The required force for melting into nanochanels is proportion to the surface tension of the melt and can be calculated using an equation from our previous reports. ${ }^{(11,12)}$ In this study, the zinc metal and indium metal in a 5:1 weight ratio were heated to $600{ }^{\circ} \mathrm{C}$, melted and evenly mixed, and then pressed into a metal plate after cooling. The zinc indium alloy was placed on the AAO template in a chamber at a pressure of $10^{-3}$ Torr and then heated above the melting point of the zinc-indium alloy, so that the zinc-indium alloy melted and covered the AAO surface. Meanwhile, a positive pressure was applied to inject the molten zinc-indium alloy into the AAO template. When the alloy solidified, the zinc-indium alloy nanowires were shaped. After the entire process was carried out, the AAO template with nanowire arrays was soaked in a $\mathrm{NaOH}$ solution for 2 h. The AAO template was removed and the finished nanowires were reveraled. The nanowires were then washed using an ultrasonic washing machine to remove the residual AAO template. Finally, an atmosphere furnace was used to heat and oxidize the nanowires into zinc-indium oxide nanowires, which were then coated with gold films as electrodes by thermal evaporation.

\subsection{Setting up a sensor testing platform}

In this research, we assembled a gas detection system, which was fed with carbon monoxide gas for detection. A pressure valve was used to control the gas entering the chamber, while a heater was used to maintain a constant temperature on the testing plate, and then a multifunctional meter (Agilent 34410A) was used to measure the resistance. The detection system is illustrated in Fig. 2. In this research, we built a zinc-indium oxide nanowire array sensing component to conduct sensitivity tests at room temperature as well as at 50 and $100{ }^{\circ} \mathrm{C}$. The measurement started with an initial 0-300 s duration, when the component was in the chamber filled with air, followed by a 300-600 $\mathrm{s}$ duration when $1000 \mathrm{ppm}$ carbon monoxide was used as the gas for detection, which reacted with the component. Then, in the 600-900 s duration, the component was exposed to air to return the chamber to its original state. 


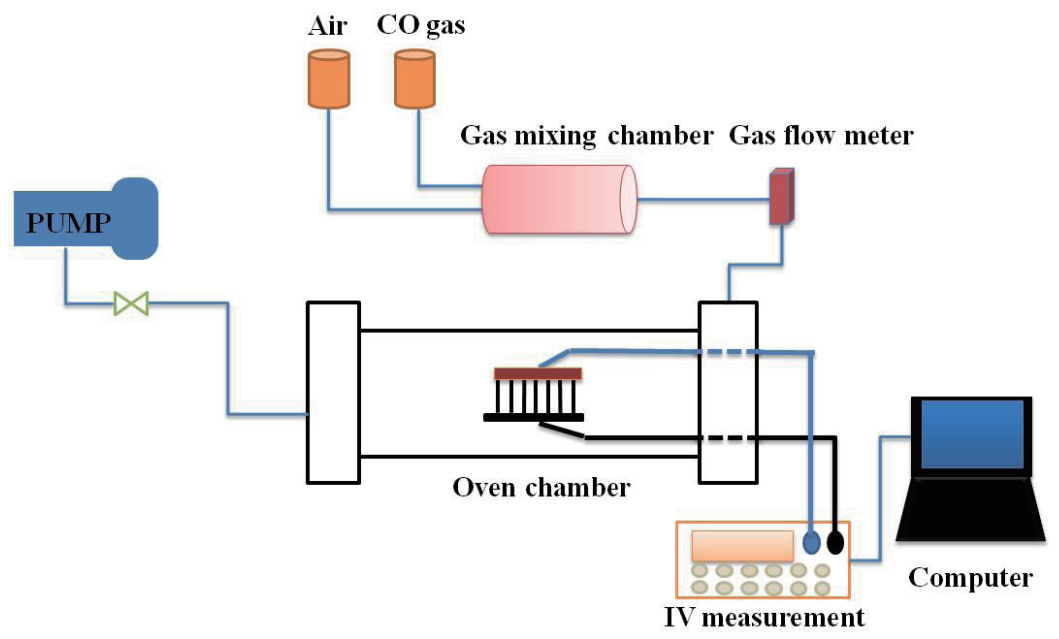

Fig. 2. (Color online) Illustration of gas sensing system.

\section{Results and Discussion}

\subsection{Production of sensing component}

SEM analysis was carried out to observe the morphologies of the AAO template and zincindium nanowires prepared in this study. In Fig. 3(a), it appears that the pore distribution was an array, with an average pore diameter of about $80 \mathrm{~nm}$, while Fig. 3(b) shows that the die-casted zinc-indium alloy was completely injected into the pores. The result of differential scanning calorimetry analysis showed that the indium-zinc alloy smelted in this research had two melting points. According to the zinc-indium phase diagram, ${ }^{(13)}$ the alloy's melting point should be $362{ }^{\circ} \mathrm{C}$, whereas the differential scanning calorimetry (DSC) experiment (Fig. 4) showed that it was around $366.7^{\circ} \mathrm{C}$, which conformed to the predefined ratio.

\subsection{Carbon monoxide testing}

In detecting gas, the resistance of the sensing material of the metal oxide semiconductor gas sensors changes, and the electronic conductivity characteristics vary to detect the gas volume. The main principle is that flammable gases detected, such as $\mathrm{CO}$, are absorbed and react with the negatively charged oxygen ions in the semiconductor. The chemical reaction causes changes in the carrier concentration, conductivity, and the resistance of the sensor. In the n-type metal oxide semiconductor, the major carriers are electrons. The resistance increases under oxygen ambient because the electrons in the conduction band are removed by the adsorbed oxygen ions [Eq. (1)]. ${ }^{(14)}$ When the sensor is under a reducing gas ambient such as $\mathrm{CO}$, the electrons are obtained from the reaction of $\mathrm{CO}$ and oxygen ions, resulting in a decrease in resistance, as described by Wang et al. ${ }^{(15)}$ Figure 5 proposed by Wang et al. shows the structural and band models of the conductive mechanism upon exposure to $\mathrm{CO}$. The reduction of oxygen ions on the semiconductor oxide surface makes the electrons trapped by oxygen return to the MOS, and this process reduces the size of the energy barrier, leading to reduced resistance.

$$
\mathrm{O}_{2}(\mathrm{gas})+e^{-}(\mathrm{MOS}) \leftrightarrow \mathrm{O}_{2}^{-} \text {(absorb) }
$$




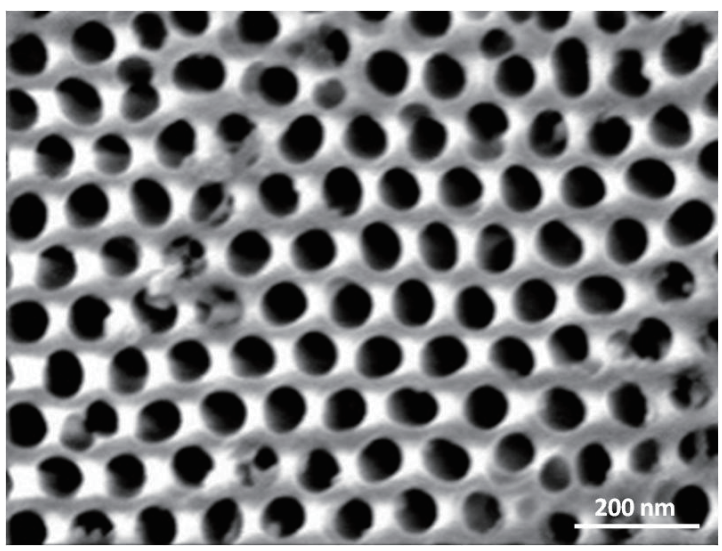

(a)

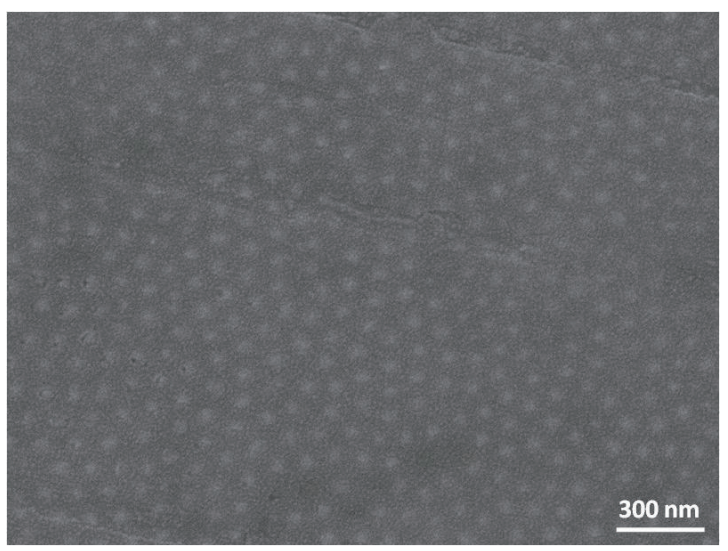

(b)

Fig. 3. (a) SEM image of AAO membrane with pores $80 \mathrm{~nm}$ in diameter and (b) zinc-indium nanowires in AAO membrane.

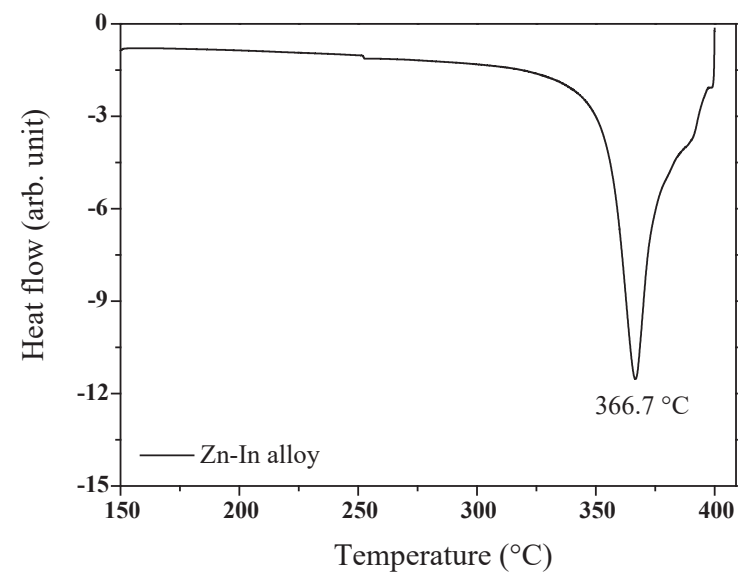

Fig. 4. DSC analysis of zinc-indium alloy.

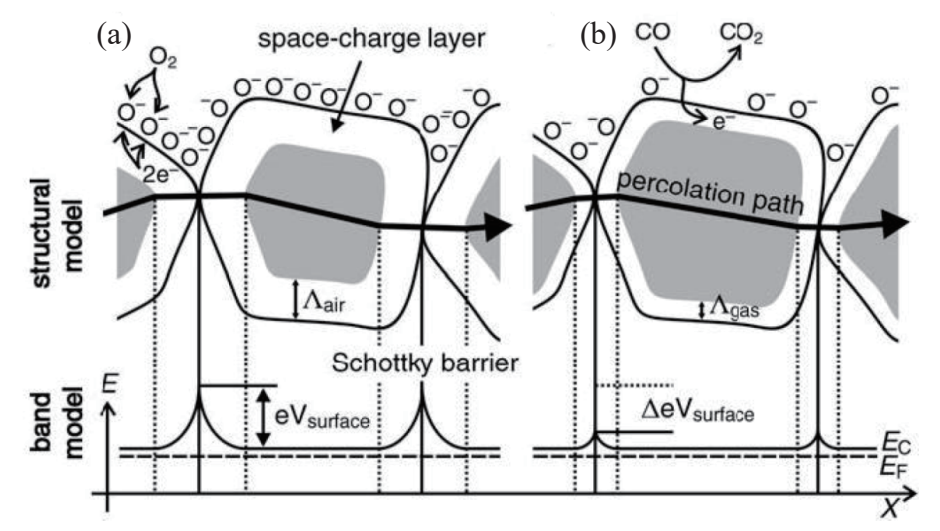

Fig. 5. Structural and band models of conductive mechanism upon exposure to reference gas (a) without and (b) with $\mathrm{CO} .{ }^{(15)}$

The gas-sensing device fabricated in this research was analyzed to yield the result shown in Fig. 6. At room temperature, when $1000 \mathrm{ppm} \mathrm{CO}$ was injected at the 300th second, the resistance showed a downward trend, but the magnitude was so small that it could be ignored. However, when the temperature was increased to $50{ }^{\circ} \mathrm{C}$, the resistance became stable, and after $\mathrm{CO}$ gas was injected, the resistance decreased instantly, generating a very obvious trough. At the 600th second, the background gas (air) was restored, and the low resistance was maintained for about $50 \mathrm{~s}$ before returning to a high-resistance state. This could be caused by the slow dissipation of $\mathrm{CO}$, which was not completed removed by the injected background gas, thus causing a persistent reaction in a period of time until the $50 \mathrm{~s}$ elapsed. When the temperature was increased to $100{ }^{\circ} \mathrm{C}$, the resistance started to fluctuate irregularly, losing the component's effectiveness as a gas sensor. At the 300th second when the mixed CO was injected, there was no obvious reaction, even though at this point there was a resistance drop; it was still within the fluctuation range of the original signal. Therefore, it was regarded as no reaction with $\mathrm{CO}$. The result of the experiment showed that a temperature of $50{ }^{\circ} \mathrm{C}$ made the zinc-indium oxide nanowire gas sensing component better in terms of reaction 


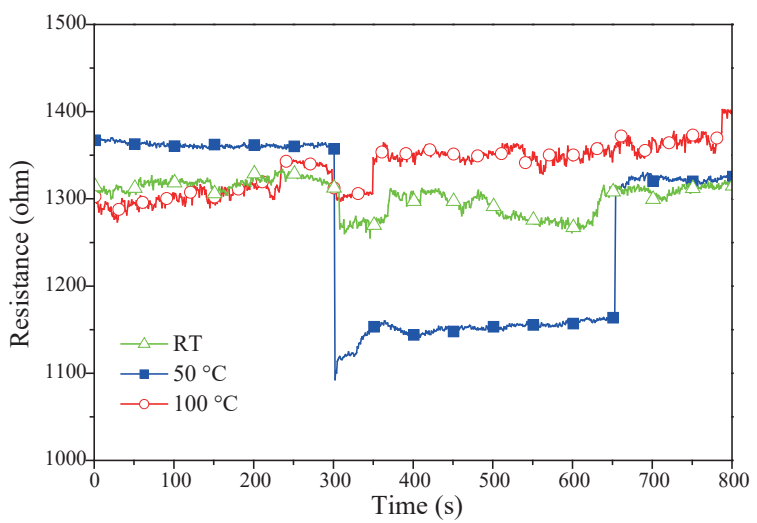

Fig. 6. (Color online) Response and recovery curves of zinc-indium oxide nanowires at room temperature, $50{ }^{\circ} \mathrm{C}$, and $100{ }^{\circ} \mathrm{C}$.

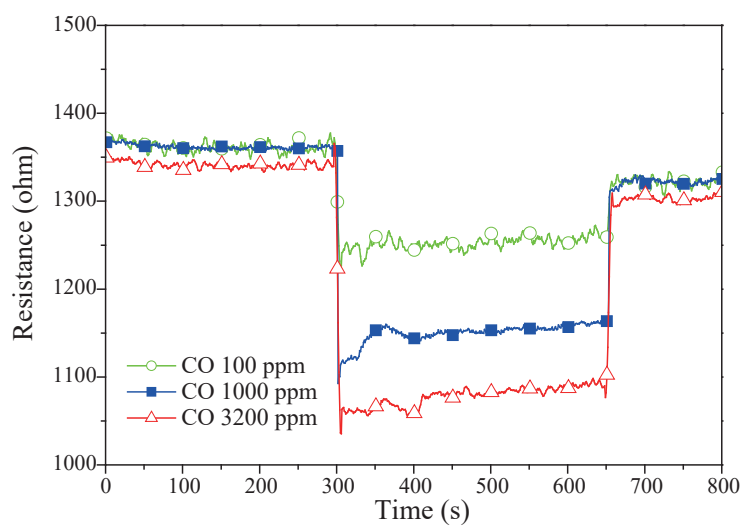

Fig. 7. (Color online) Response and recovery curves of zinc-indium oxide nanowires at different concentrations of $\mathrm{CO}$ at $50{ }^{\circ} \mathrm{C}$.

effect and sensitivity. Figure 7 shows the sensor's responses to various CO concentrations (100, 1000 , and $3200 \mathrm{ppm}$ ) at $50^{\circ} \mathrm{C}$. The result indicated that more CO reacting with MOS would lead to a higher reduction in resistance. It also revealed that the zinc-indium nanowire gas sensor could work at $\mathrm{CO}$ concentrations as low as $100 \mathrm{ppm}$.

\section{Conclusions}

In this research, we successfully used a vacuum die casting method to produce a zinc-indium oxide nanowire gas sensing component. The component was tested at room temperature, 50 and $100{ }^{\circ} \mathrm{C}$, and it was concluded that the operating temperature of $50{ }^{\circ} \mathrm{C}$ gave the sensor a better reaction effect and sensitivity. Compared with other temperatures, $50{ }^{\circ} \mathrm{C}$ is the most ideal operating temperature. Results of sensing various $\mathrm{CO}$ concentrations showed that the zinc-indium oxide nanowire gas sensor still had good response to lower CO concentration (100 ppm).

\section{References}

1 K. Galatsis, L. Cukrov, W. Wlodarski, P. McCormick, K. Kalantar, E. Comini, and G. Sberveglieri: Sens. Actuators, B 93 (2003) 562.

2 G. Korotcenkov: Mater. Sci. Eng. B 139 (2007) 1.

3 M. Shahabuddin, A. Sharma, J. Kumar, M. Tomar, A. Umar, and V. Gupta: Sens. Actuators, B 194 (2014) 410.

4 E. Comini: Anal. Chim. Acta 568 (2016) 28.

5 D. Zhang, Z. Liu, C. Li, T. Tang, X. Liu, S. Han, B. Lei, and C. Zhou: Nano Lett. 4 (2004) 1919.

6 A. Renitta and K. Vijayalakshmi: Sens. Actuators, B 237 (2016) 912.

7 H. S. Gu, Z. Wang, and Y. M. Hu: Sensors 12 (2012) 5517.

8 R. R. Zhou, G. F. Hu, R. M. Yu, C. F. Pan, and Z. L. Wang: Nano Energ. 12 (2015) 588.

9 P. Dhivya, A. K. Prasad, and M. Sridharan: Int. J. Hydrogen Energ. 37 (2012) 18575.

10 A. C. Nwanya, P. R. Deshmukh, R. U. Osuji, M. Maaza, C. D. Lokhande, and F. I. Ezema: Sens. Actuators, B $206(2015) 671$.

11 C. G. Kuo, H. Chang, L. R. Hwang, S. Hor, J. S. Chen, G. Y. Liu, and S. C. Cheng: Electron. Mater. Lett. 9 (2013) 481.

12 C. G. Kuo, J. H. Chen, and Y. H. Liu: Int. J. Photoenergy 2014 (2014) 302075.

13 T. B. Massalski: Binary Alloy Phase Diagrams (American Society for Metals, Ohio, 1990).

14 X. H. Peng: Nanowires-Recent Advances (In Tech, Rijeka, 2012) Chap. 1.

15 C. X. Wang, L. W. Yin, L. Y. Zhang, D. Xiang, and R. Gao: Sensors 10 (2010) 2088. 